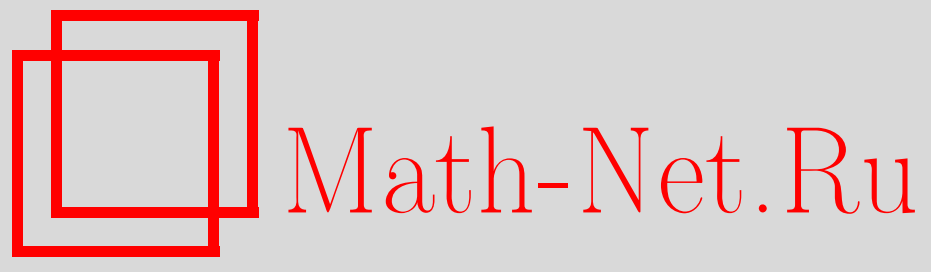

У. Х. Каримов, Д. Реповш, Гавайские группы топологических пространств, УМН, 2006, том 61, выпуск 5, 185186

DOI: https://doi.org/10.4213/rm3504

Использование Общероссийского математического портала Math-Net.Ru подразумевает, что вы прочитали и согласны с пользовательским соглашением http://www .mathnet.ru/rus/agreement

Параметры загрузки:

IP: 54.92 .164 .108

26 апреля 2023 г., 14:39:08

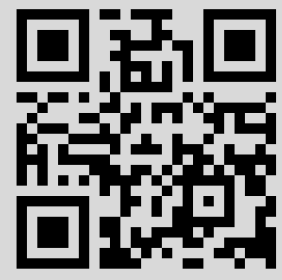




\section{Гавайские группы топологических пространств}

\section{У. Х. Каримов, Д. Реповш}

$n$-мерной Гавайской серъгой $(n=0,1,2, \ldots)$ называется следующее подпространство $(n+1)$-мерного евклидова пространства $\mathbb{R}^{n+1}$ :

$$
\mathscr{H}^{n}=\left\{\bar{x}=\left(x_{0}, x_{1}, \ldots, x_{n}\right) \in \mathbb{R}^{n+1} \mid\left(x_{0}-1 / k\right)^{2}+\sum_{i=1}^{n} x_{i}^{2}=(1 / k)^{2}, k \in \mathbb{N}\right\} .
$$

Точку $\theta=(0,0, \ldots)$ будем считать отмеченной точкой $\mathscr{H}^{n}$.

$n$-мерное Гавайское множество пространства $X$ с отмеченной точкой $x_{0}$ определяется как множество гомотопических классов $[f]$ отображений $f:\left(\mathscr{H}^{n}, \theta\right) \rightarrow\left(X, x_{0}\right)$. Обозначим его символом $\mathscr{H}_{n}\left(X, x_{0}\right)$. При $n \geqslant 1$ в $\mathscr{H}_{n}\left(X, x_{0}\right)$ естественно определяется групповая операция, порожденная группами $\pi_{n}\left(X, x_{0}\right)$. Группы $\mathscr{H}_{n}\left(X, x_{0}\right)$ (множества $\left.\mathscr{H}_{0}\left(X, x_{0}\right)\right)$ являются гомотопическими инвариантами в категории всех топологических пространств с отмеченными точками.

Теорема 1. Если пространство $X$ локально $п$-связно в точке $x_{0}$ и удовлетворлет первой аксиоме счетности, то группа $\mathscr{H}_{n}\left(X, x_{0}\right)$ изоморфна слабому прямому произведению $\prod_{i=0}^{\infty} G_{i}$, в котором все $G_{i}=\pi_{n}\left(X, x_{0}\right) \ldots$.

Доказательство. Рассмотрим любое отображение $f:\left(\mathscr{H}^{n}, \theta\right) \rightarrow\left(X, x_{0}\right)$. Так как $X$ локально $n$-связно в $x_{0}$, то существует окрестность $V_{x_{0}}$ такая, что вложение $V_{x_{0}} \subset X \quad n$-тривиально. Так как $f$ непрерывно, то существует натуральное число $K$ такое, что $S_{k}^{n} \subset f^{-1}\left(V_{x_{0}}\right)$ для $k>K$. Поэтому все отображения $\left.f\right|_{S_{k}^{n}}$ являются $n$-тривиальными при $k>K$. Определим отображение $\varphi: \mathscr{H}_{n}\left(X, x_{0}\right) \rightarrow \prod_{i=1}^{\infty} G_{i}$ следующим образом: $\varphi([f])=\left(\left[\left.f\right|_{S_{1}^{n}}\right],\left[\left.f\right|_{S_{2}^{n}}\right],\left[\left.f\right|_{S_{3}^{n}}\right], \ldots,\left[\left.f\right|_{S_{K}^{n}}\right], e, e, \ldots\right) \in \prod_{i=0}^{\infty} G_{i}$. Очевидно, что $\varphi$ - сюръективное отображение. Докажем, что $\varphi$ инъективно. Для этого рассмотрим два отображения $f$ и $g$ такие, что $\varphi(f)=\varphi(g)$. Так как пространство $X$ локально $n$ связно и удовлетворяет первой аксиоме счетности, то существует счетная вложенная система $U_{i}$ окрестностей точки $x_{0}$ такая, что все вложения $U_{i+1} \subset U_{i}$ гомотопически $n$-тривиальны. Существует возрастающая система натуральных чисел $\left\{K_{i}\right\}_{i \in \mathbb{N}}$ такая, что $\operatorname{Im}\left(\left.f\right|_{S_{k}^{n}}\right) \cup \operatorname{Im}\left(\left.g\right|_{S_{k}^{n}}\right) \subset U_{m+1}$ при всех $k>K_{m}$. Для $k \leqslant K_{1}$ выберем любую гомотопию относительно точки $\theta$, которая связывает отображение $\left.\left.f\right|_{S_{k}^{n}} \mathrm{c} g\right|_{S_{k}^{n}}$ (это можно сделать так как $\varphi(f)=\varphi(g))$. Для $k$ таких, что $K_{1}<k \leqslant K_{2}$, выберем в множестве $U_{1}$ любую гомотопию между $\left.f\right|_{S_{k}^{n}}$ и $\left.g\right|_{S_{k}^{n}}$. Вообще, для $k$ таких, что $K_{m}<k \leqslant K_{m+1}$, выберем любую гомотопию, связывающую $\left.f\right|_{S_{k}^{n}}$ с $\left.g\right|_{S_{k}^{n}} ^{n}$ в множестве $U_{m}$. В результате мы получим гомотопию относительно точки $\theta$ между $f$ и $g$, т.е. $\varphi$ инъективно.

Теорема 2. Если пространство $X$ имеет счетную систему окрестностей в точке $x_{0}$ и группы $\mathscr{H}_{n}\left(X, x_{0}\right)$ (множества $\left.\mathscr{H}_{0}\left(X, x_{0}\right)\right)$ счетны, тогда $X$ локально $n$-связно в точке $x_{0}$.

ДокАЗАтельство. Предположим, что $X$ не локально $n$-связно в точке $x_{0}$. Тогда существует вложенная система открытых окрестностей $V_{i}$ точки $x_{0}$ такая, что вложения $V_{i} \subset V_{1}$ существенны в размерности $n$ и $\bigcap_{i=1}^{\infty} V_{i}=x_{0}$. Выберем для каждого $i$ отображение $f_{i}: S^{n} \rightarrow V_{i}$, композиция которого с вложением $V_{i} \subset V_{1}$ гомотопически существенна. Далее, каждой последовательности нулей и единиц $\sigma=\left(\sigma_{1}, \sigma_{2}, \sigma_{3}, \ldots\right)$, $\sigma_{i}=0$ или 1 , очевидно, соответствует отображение $f_{\sigma}:\left(\mathscr{H}^{n}, \theta\right) \rightarrow\left(X, x_{0}\right)$. Рассмотрим две такие последовательности $\sigma$ и $\sigma^{\prime}$, что $\sigma_{i} \neq \sigma_{i}^{\prime}$ для бесконечного множества индексов. Отображение $f_{\sigma}$ гомотопически не эквивалентно $f_{\sigma^{\prime}}$. Действительно, предположим, что они гомотопически эквивалентны и пусть гомотопия $H:\left(\mathscr{H}^{n}, \theta\right) \times I \rightarrow$ $\left(X, x_{0}\right)$ соединяет $f_{\sigma}$ и $f_{\sigma^{\prime}}$. Так как $H(\theta \times I)=x_{0} \in V_{1}$, то существует $K$ такое,

Работа выполнена при поддержке Министерства образования, науки и спорта Республики Словения по программе № 0101-509. 
что $H\left(S_{k}^{n} \times I\right) \subset V_{1}$ при $k>K$. Так как $\sigma_{i} \neq \sigma_{i}^{\prime}$ для бесконечного числа индксов, то существует $k_{0}>K$, для которого $\sigma_{k_{0}} \neq \sigma_{k_{0}}^{\prime}$. Тогда одно из отображений $\left.f_{\sigma}\right|_{S_{k_{0}}^{n}}: S_{k_{0}}^{n} \rightarrow V_{k_{0}} \rightarrow V_{1}$ или $\left.f_{\sigma^{\prime}}\right|_{S_{k_{0}}^{n}}: S_{k_{0}}^{n} \rightarrow V_{k_{0}} \rightarrow V_{1}$ гомотопически существенно, а другое гомотопически постоянно. Это противоречит тому, что $H\left(S_{k_{0}}^{n} \times I\right) \subset V_{1}$. Поэтому $f_{\sigma}$ и $f_{\sigma^{\prime}}$ гомотопически неэквивалентны.

Так как множество всех $\sigma$, которые отличаются друг от друга на бесконечном множестве индексов, несчетно, то $\mathscr{H}_{n}\left(X, x_{0}\right)$ несчетно. Это противоречит предположениям теоремы 2.

СледСтвиЕ 1. Связное компактное метрическое пространство $X$ есть континуум Пеано в том и только том случае, когда множество $\mathscr{H}_{0}\left(X, x_{0}\right)$ счетно в каждой точке $x_{0}$ пространства $X$.

СлеДСтвиЕ 2. Конечномерное компактное метрическое пространство $X$ есть ANR в том и только том случае, когда группы $\mathscr{H}_{n}\left(X, x_{0}\right)$ счетны для всех $n$ и всех точек $x_{0} \in X$.

СледСтвиЕ 3. Конечномерное компактное метрическое пространство $X$ есть AR тогда и только тогда, когда $\mathscr{H}_{n}\left(X, x_{0}\right)=e$ при всех $n$ и во всех точках $x_{0}$ пространства $X$.

ЗАмечание 1 . Существует стягиваемый компакт $X$ такой, что $\mathscr{H}_{1}(X, *) \neq e$ для некоторой точки $*$.

Конус над 1-мерной Гавайской серьгой $C\left(\mathscr{H}^{1}, \theta\right)$ является таким пространством (* - любая внутренняя точка сегмента $C(\theta))$. Конус $C\left(\mathscr{H}^{1}, \theta\right)$ не локально 1-связен в $*$, поэтому $\mathscr{H}_{1}\left(C\left(\mathscr{H}^{1}, \theta\right), *\right) \neq e$.

ЗАмечание 2 (К. Эда). Существует локально 1-связный во всех точках компакт $X$ такой, что группа $\mathscr{H}_{1}(X, *)$ несчетна во всех его точках. Например, надстройка $\Sigma C$ над компактом Кантора $C$ является таким пространством.

ЗАмечание 3. Существует локально 2-связный континуум Пеано $X$ такой, что группы $\mathscr{H}_{2}(X, *)$ несчетны во всех точках $*$.

Букет 2-мерной сферы и 1-мерной Гавайской серьги является таким пространством.

Теорема 3. Существует нестягиваемый клеточно-подобный компакт $X$ такой, что для всех $n$ и всех точек $x_{0} \in X$ группа $\mathscr{H}_{n}\left(X, x_{0}\right)$ тривиальна.

ДокАзАтельство. Рассмотрим счетный компактный букет сфер возрастающей размерности $\bigvee_{i=1}^{\infty} S^{i}$ с отмеченной точкой $\theta$. Пусть $C\left(\bigvee_{i=1}^{\infty} S^{i}\right)$ есть конус над букетом $\bigvee_{i=1}^{\infty} S^{i}$ с вершиной $a$ и с основанием, которое мы отождествим с $\bigvee_{i=1}^{\infty} S^{i}$. Пусть $\theta \in \bigvee_{i=1}^{\infty} S^{i} \subset C\left(\bigvee_{i=1}^{\infty} S^{i}\right)$ будет отмеченной точкой конуса. Пусть $X_{1}$ и $X_{2}$ - две копии конуса $C\left(\bigvee_{i=1}^{\infty} S^{i}\right)$ с вершинами $a_{1}, a_{2}$ и с отмеченными точками $\theta_{1}, \theta_{2}$ соответственно. Пусть $X$ есть одноточечное объединение пространств $X_{1}$ и $X_{2}$ относительно точек $\theta_{1}$ и $\theta_{2}$. Очевидно, $X$ удовлетворяет всем условиям теоремы.

Вопрос. Пусть $P$ и $P^{*}$ суть одноточечные компактификации счетных полиэдров точками $\theta$ и $\theta^{*}$ соответственно. Пусть $f:(P, \theta) \rightarrow\left(P^{*}, \theta^{*}\right)$ есть такое непрерывное отображение, что $\mathscr{H}_{n}(f): \mathscr{H}_{n}(P, \theta) \rightarrow \mathscr{H}_{n}\left(P^{*}, \theta^{*}\right)$ является изоморфизмом для всех $n$. Верно ли, что $f$ есть гомотопическая эквивалентность?

\section{У. X. Каримов (U. Kh. Karimov)}

Институт математики АН Республики Таджикистан, Душанбе, Таджикистан

E-mail: umed-karimov@mail.ru

\section{Д. Реповш (D. Repovš)}

Institute of Mathematics, Physics and Mechanics,

University of Ljubljana, Ljubljana, Slovenia

E-mail: dusan.repovs@uni-lj.si
Представлено В. М. Бухштабером Принято редколлегией 24.07.2006 